\title{
A New Coding Metasurface for Wideband RCS Reduction
}

\author{
Liaori JIDI, Xiangyu CAO, Yao TANG, Siming WANG, Yi ZHAO, Xuewen ZHU \\ Information and Navigation College, Air Force Engineering University, Xi'an 710077, China \\ jidiliaorikdy@163.com
}

Submitted September 1, 2017 / Accepted April 16, 2018

\begin{abstract}
In this paper, two novel artificial magnetic conductor (AMC) structures are designed to realize 180 degrees of phase difference in a wideband frequency. These two AMC structures are encoded as unit " 0 " and unit " 1 ", respectively. By using Simulated Annealing algorithm, the coding sequences of the coding metasurface can be designed, so that the radar cross section (RCS) reduction can be realized as well. Compared with the metallic surface, the simulation and measurement results of this presented coding metasurface indicate that this coding metasurface can reduce RCS significantly under normally incident electromagnetic (EM) waves from $7 \mathrm{GHz}$ to $20 \mathrm{GHz}$, so the bandwidth is $96.3 \%$, and the RCS under obliquely incident waves also can be dramatically reduced as well. Furthermore, the RCS reduction of this coding metasurface is better than that of traditional chessboard surface.
\end{abstract}

\section{Keywords}

Coding metasurface, wideband, RCS reduction

\section{Introduction}

Metasurfaces are ultrathin planar artificial structures composed of periodic or non-periodic unit cells which are much smaller than wavelength [1-4]. By introducing abrupt phase shift to the surfaces, metasurfaces can perform unique electromagnetic (EM) properties, such as anomalous refection and refraction, perfect lensing, invisibility cloaking and polarization conversion [5], [6], which have not yet been found in nature, so they have attracted much research attention since their first presentation. One of the most important applications of metasurfaces is EM cloaking which can be used to reduce the radar cross section (RCS) of metallic or dielectric targets [7]. Metasurfaces can be considered as effective two-dimensional structures, and many researchers have focused on the properties of metasurfaces that can control reflected and transmitted waves [8].

With the urgent demand of stealth technology for platforms, researchers have paid a lot of attention in radar cross section (RCS) reduction. There are two main methods to reduce RCS. One is to use perfect metamaterial absorber (PMA) with near-unity absorptivity and ultrathin structure, which was originally proposed by Landy et.al in 2008 [9]. From that time and ever since, researchers have proposed many different kinds of PMA structures to achieve high absorption in wideband frequency. The other way is to use planar configuration. It means that with the different combinations of artificial magnetic conductors (AMC), phase cancellation can be achieved [10], so that the RCS can be reduced as well. Many researchers and scientists have combined various kinds of AMC structures to form chessboard surfaces to reduce RCS in wide frequency band [11-13]. For example, M. Panquay proposed a novel planar configuration based on thin AMC structure to achieve low RCS [14], and Wengang Chen proposed several kinds of chessboard surfaces to achieve wideband and dual-band RCS reduction in low frequency [11], [12].

In recent three years, the intriguing properties of coding metamaterials and metasurfaces have captivated the interests and imaginations of leading scientists [10], [13-17]. In [10], Tie Jun Cui came up with the concept of coding metamaterial and gave examples of 1-bit and 2-bit coding metamaterials. In the following few years, Tie Jun Cui and his colleagues have designed different kinds of coding metasurfaces. For example, they designed an anisotropic coding metasurface working in terahertz frequency [15], and they also designed a transmission-type coding metasurface working in terahertz frequency [18]. Shuo Liu proposed frequency-dependent and dual-functional coding metasurfaces at terahertz frequencies in [19]. Si Jia $\mathrm{Li}$ proposed an ultra-broadband reflective metamaterial with RCS reduction using coding methods last year [7], and Yi Zhao designed a broadband diffusion metasurface by tailoring a single anisotropic element [20]. The coding metasurfaces can manipulate the EM waves significantly. In the designs of coding metasurfaces, the coding sequences of " 0 " and " 1 " elements are introduced to achieve various functionalities of EM scattered fields, such as anomalous reflection and scattered beam diffusion, to reduce RCS [20].

The main objective of this paper is to design two AMC structures whose phase difference can reach 180 degrees in a wideband frequency, and these two AMC 
structures can both realize total reflection. In this paper, two AMC structures are designed to realize 180 degrees of reflection phase difference in a wideband frequency. These two AMC structures are encoded as unit " 0 " and unit " 1 ", respectively. By using Simulated Annealing algorithm, the coding sequences of coding metasurface can be designed, so that the metasurface can redirect the incident EM waves into different directions as many as possible and the RCS of this coding metasurface can be reduced as well. Compared with metallic surface, the simulation and measurement results of this presented coding metasurface indicate that this coding metasurface can significantly realize RCS reduction under normally incident EM waves from $7 \mathrm{GHz}$ to $20 \mathrm{GHz}$, and the RCS under obliquely incident waves also can be dramatically reduced as well. Furthermore, the RCS reduction of this coding metasurface is better than that of traditional chessboard surface.

This paper is organized as follows. The design and simulation of unit cells of AMC structures are introduced in Sec. 2. The analysis and optimized coding sequences of two AMC structures are presented in Sec. 3. Meanwhile, the simulation comparison between the proposed coding metasurface and conventional chessboard metasurface consisted of the same unit cells is given in Sec. 4, and the measurement result is given in this section as well. Finally, the work is concluded in Sec. 5.

\section{Design of Unit Cells}

One of the most important characteristics of AMC is the phase variation of its reflection coefficient [12]. Generally, the reflection phase of AMC structure is 0 degree at resonant frequency, while the reflection phase of perfect electric conductor (PEC) is 180 degrees constantly [11]. Meanwhile, the reflection magnitude of AMC structure with a lossless ground is unity. Based on the analysis of conventional chessboard surface, the phase difference of the proposed two AMC structures is aimed to reach 180 degrees as approximate as possible to achieve low RCS. In this paper, two AMC structures which are composed of circular loop patch and substrate are designed because of their simplicity of design and proper characteristics. The AMC with higher reflection phase is encoded as unit " 1 ", while the AMC with lower reflection phase is encoded as unit " 0 ".

Consider a unit cell of split circular loop, as illustrated in Fig. 1. The detailed geometrical parameters are: $p=10 \mathrm{~mm}, r=4.25 \mathrm{~mm}, w=2 \mathrm{~mm}$ and $h=3 \mathrm{~mm}$. The size of unit cell for the coding metasurface is, in principle, from $\lambda / 8$ to $\lambda / 4$; while the size of unit cell for the reflectarray or transmitarrays is typically over half wavelength (not less than $\lambda / 3$ ) [16]. The size of the unit cell we choose in this paper is $0.26 \lambda_{0} \times 0.26 \lambda_{0}\left(\lambda_{0}\right.$ represents free space wavelength at the first frequency point where the phase difference of these two proposed AMC structures reach 180 degrees), and $\alpha$ is a variable.

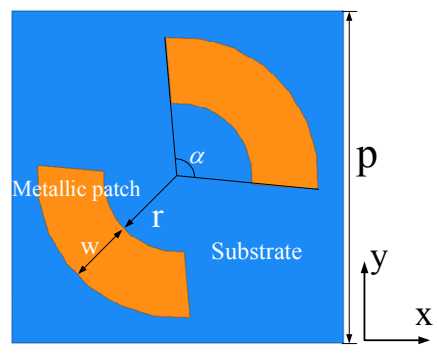

Fig. 1. Unit cell.

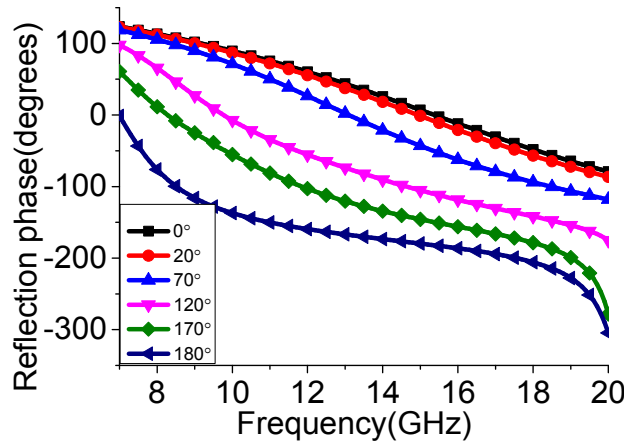

Fig. 2. Co-polarized reflection phases under different $\alpha$ values for both normally x-polarized and y-polarized incident EM waves.

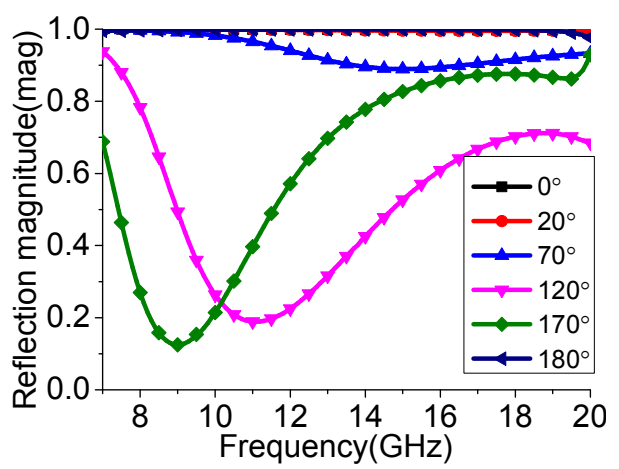

Fig. 3. Co-polarized reflection magnitudes under different $\alpha$ values for both normally x-polarized and y-polarized incident EM waves.

The split circular loop is located on the top of a substrate with a thickness of $3 \mathrm{~mm}$ and a dielectric constant of 2.65 , and the bottom surface of the substrate is PEC. This structure is simulated using Ansoft HFSS. The reference plane of reflection phase is the upper surface of the AMC structure, and the reflection coefficient characteristics of the infinite array of this unit cell versus the angle $\alpha$ under normally incident EM waves are illustrated in Fig. 2 and Fig. 3.

Figures 2 and 3 show that the co-polarized reflection coefficient characteristics for both normally $\mathrm{x}$ - and $\mathrm{y}$ polarized incidences are the same, which is caused by the symmetrical structure of this unit cell. Figure 2 indicates that the 180 degrees of phase difference can be reached if the $\alpha$ values are properly chosen. It is indicated from Fig. that the incident EM waves can be totally reflected under certain $\alpha$ angles. The design purpose of this paper is 


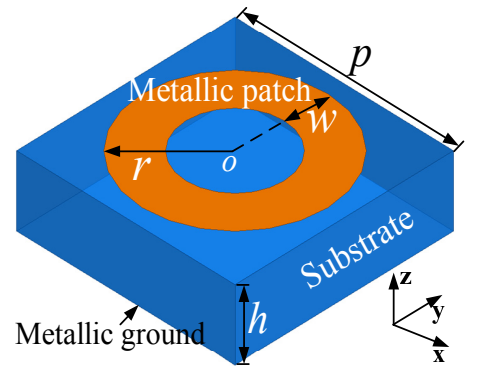

(a)

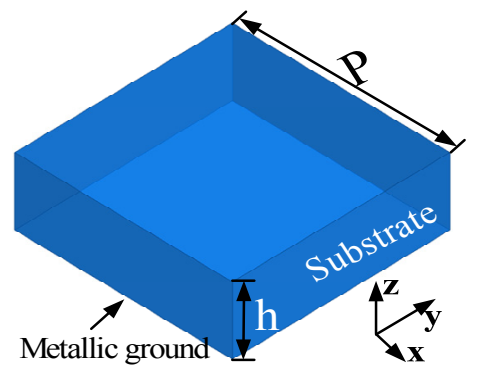

(b)

Fig. 4. Unit cell of (a) AMC1 structure and (b) AMC2 structure.

to realize 180 degrees of phase difference and fully reflection of incident waves. Guided by this principle, two different unit cells with $\alpha$ values of 180 degrees and 0 degree, respectively, can be designed based on this split circular loop. The split circular loop with 180 degrees of $\alpha$ value is degenerated to a circular loop and the split circular loop with 0 degree of $\alpha$ value is degenerated to a substrate.

The proposed unit cell of AMC1 is illustrated in Fig. 4(a) and its detailed dimensions are as follows. $p=10 \mathrm{~mm}, r=4.25 \mathrm{~mm}, w=2 \mathrm{~mm}$ and $h=3 \mathrm{~mm}$. As previously demonstrated in Fig. 2, the resonant frequency occurs at the frequency where the reflection phase is 0 degree, so it can be seen that the resonant frequency of AMC1 occurs at $7 \mathrm{GHz}$ and the magnitude of reflection coefficient can maintain unity at the whole operation bandwidth.

The proposed unit cell of AMC2 structure based on substrate is illustrated in Fig. 4(b). Its detailed dimensions are as follows. $p=10 \mathrm{~mm}$ and $h=3 \mathrm{~mm}$. It can be seen from Fig. 2 that its resonant frequency occurs at $14.9 \mathrm{GHz}$ and its magnitude also can maintain unity at the whole operation frequency.

Originally, the coding metasurface is the extending of conventional chessboard surface, so the characteristics analysis of coding metasurface can be based on that of chessboard surface. An approximate expression of RCS reduction of chessboard, compared with that of PEC, was introduced in [11]

$$
\text { RCS reduction }=10 \log \left[\frac{A_{1} \mathrm{e}^{\mathrm{j} P_{1}}+A_{2} \mathrm{e}^{\mathrm{j} P_{2}}}{2}\right]^{2} \mathrm{~dB} .
$$

$A_{1}$ and $A_{2}$ are the reflection coefficient magnitudes of two AMC structures, and $P_{1}$ and $P_{2}$ are their reflection phases,

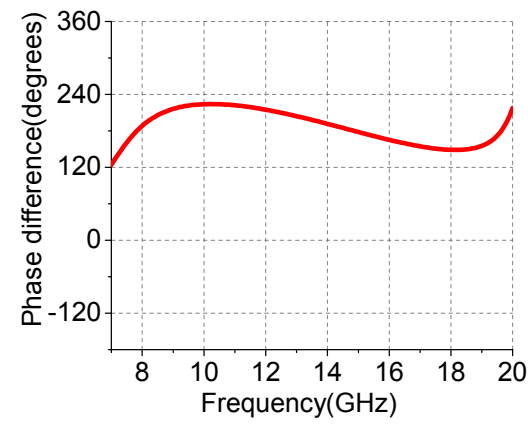

Fig. 5. Phase difference between AMC1 and AMC2.

respectively. From Fig. 3, it can be seen that both the magnitudes of two AMC structures can be unity, so the RCS reduction of chessboard surface versus the phase difference can be expressed by (2)

$$
\text { RCS reduction }=10 \log \left[\frac{1+\mathrm{e}^{\mathrm{j} \Delta}}{2}\right]^{2} \mathrm{~dB} .
$$

$\Delta$ is the phase difference between these two AMC structures. In the ideal case of infinite chessboard surface, the $10-\mathrm{dB}$ RCS of a chessboard occurs within the $\left(180^{\circ} \pm 37^{\circ}\right)$ angular range of phase difference between these two AMC structures [11]. The phase difference between these two AMC structures is illustrated in Fig. 5.

Figure 5 makes it clear that the phase difference between these two AMC structures can reach $\left(180^{\circ} \pm 37^{\circ}\right)$ in the frequency band of $7.1 \mathrm{GHz}-20 \mathrm{GHz}$, so these two proposed AMC structures can be used as unit " 0 " and " 1 " in the design of coding metasurface.

\section{Simulation and Discussion}

Because the phase difference between these two proposed AMC structures can reach $\left(180^{\circ} \pm 37^{\circ}\right)$, the AMC1 based on circular loop can be encoded as unit " 0 " and the AMC2 based on substrate can be encoded as unit " 1 ". In order to satisfy the periodic boundary in the simulation, a lattice which contains $4 \times 4$ elements of AMC1 or AMC2 is introduced in this paper, as illustrated in Fig. 6. The goal of this paper is to design a surface which can redirect the scattering energy into different directions as many as possible to minimize directional reflection. Guided by this principle, the reflection phase from each part of the surface

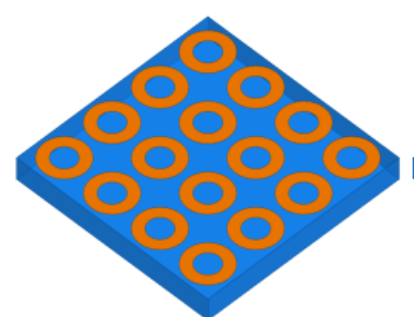

(a)

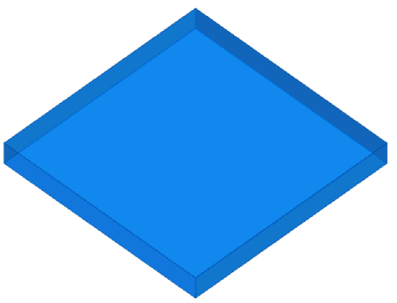

(b)
Fig. 6. Unit lattice of the proposed coding metasurface: (a) unit " 0 " and (b) unit " 1 ". 
should be distorted as much as possible rather than in an equal shifting manner. Generally, the bigger the size of the metasurface is, the better the diffusion effect can be obtained. However, considering the time cost of the simulation procedure and the overall scale of the metasurface, an array which contains $7 \times 7$ lattices of opposite reflection phases ( 0 and 180 degrees) but equal reflection magnitude under normally incident waves is chosen in this paper to illustrate the design method.

To make a better understanding of coding metasurface, a coding matrix $\boldsymbol{M}$ is introduced in this paper to describe the reflective coding metasurface

$$
\boldsymbol{M}=\left|\begin{array}{ccc}
m_{11} & \cdots & m_{17} \\
\vdots & \ddots & \vdots \\
m_{71} & \cdots & m_{77}
\end{array}\right|, m_{i j}=0 \text { or } 1 .
$$

The distribution of lattice " 0 " or lattice " 1 " can be arbitrary. According to the array theory, the scattered farfield function can be expressed as (4) under normal incidences [21]

$$
f(\theta, \varphi)=E F \cdot A F .
$$

$E F$ is the pattern function of a lattice, and $A F$ is the array factor. In this paper, $E F$ is assumed to be fixed, and $A F$ can be expressed as (5)

$A F=$

$\sum_{m=1}^{7} \sum_{n=1}^{7} \exp \{\mathrm{j}\{\varphi(m, n)+k D \sin \theta[(m-0.5) \cos \varphi+(n-0.5) \sin \varphi]\}\}$.

$\theta$ and $\varphi$ are the elevation and azimuth angles of an arbitrary observe directions, respectively, $\varphi(m, n)$ is the reflection phase of each lattice and it can be translated into " 0 " or "1" by $0 \times 180$ or $1 \times 180, k$ is the propagation constant, and $D$ is the distance between two adjacent lattices.

The coding metasurface manipulates the EM waves through different coding matrixes $\boldsymbol{M}$. For example, scattered far-field patterns of different coding metasurfaces based on the different coding matrixes are given in Fig. 8.

Using (5), it can be analyzed that the normally incident EM waves can be scattered as one single main beam if all the elements of coding matrix are the same. From Fig. 7(b) and (c), it can be seen that the normally incident EM waves can be scattered into two main beams and four main beams under these two different coding matrixes, respectively, and case (c) is the conventional chessboard surface. The coding matrix of case (d) is the random matrix and it can be seen that the normally incident EM waves are scattered into several main beams.

In order to reduce RCS of coding metasurface, the coding metasurface has to scatter the normally EM incident waves into different main beams as many as possible, and this can be achieved by using Simulated Annealing algorithm because of its merits of simple description and high efficiency. It is a method for local searching and begins

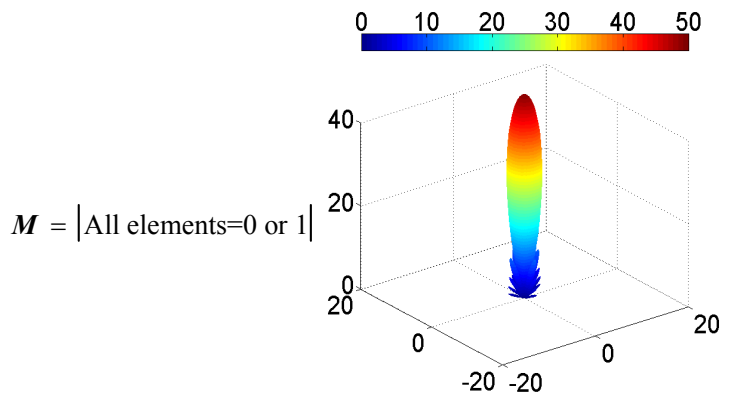

(a)

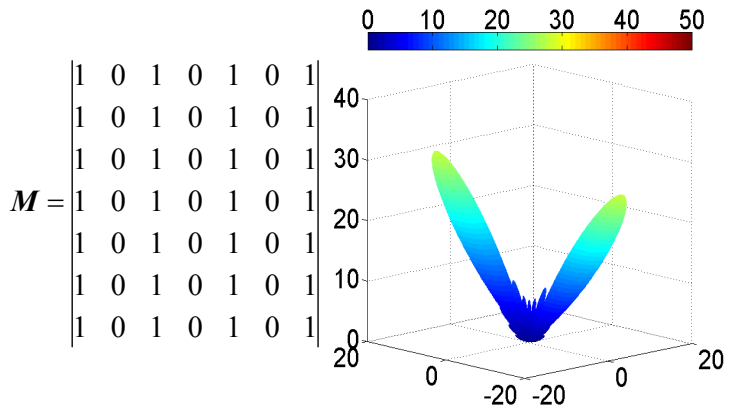

(b)

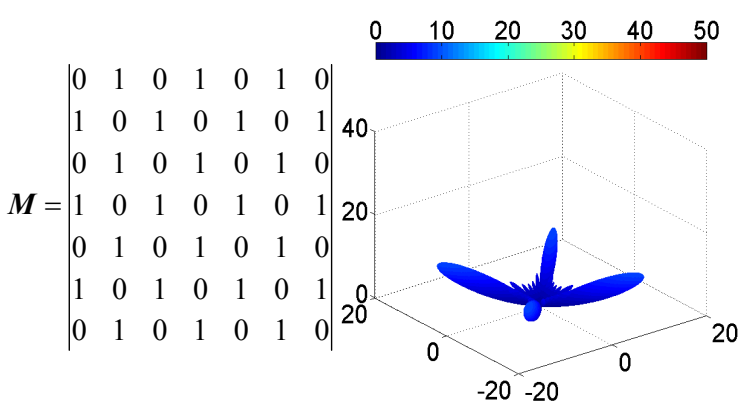

(c)

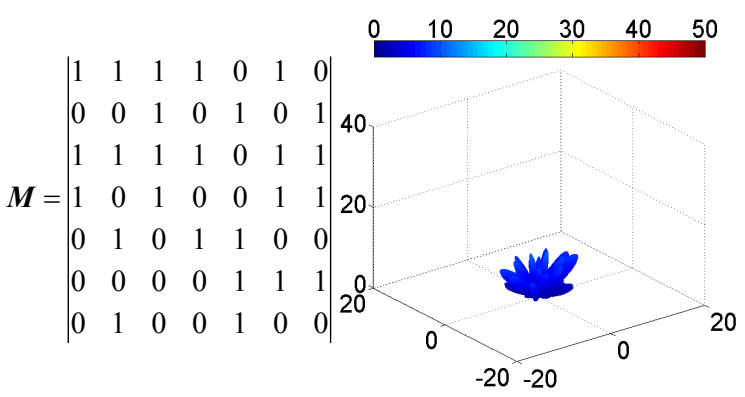

(d)

Fig. 7. Different coding matrixes and their corresponding 3-D scattered far-field pattern.

with an initial solution that is randomly modified in an iterative process. This algorithm was inspired by the annealing procedure in metallurgy, a technique involving heating and cooling control of a material to increase the size of its crystals and reduce its defects. Heating and cooling the material affects both the temperature and the thermodynamic free energy. The main parameters of Simulated Annealing algorithm are the initial temperature $T$, the decreasing rate in each iteration $\kappa$, the final temperature $T_{\mathrm{f}}$, the number of iterations $I$ and the merit function. In this 
paper, the parameters of $T, T_{\mathrm{f}}, \kappa$ and $I$ are set as $100,0,0.9$, and 3000, respectively. Let the initial coding matrix has 25 elements of " 0 " and the rest elements of " 1 ".

In order to realize low RCS performance, the optimized coding matrix should lead the desired scattering field with smallest maximum value. So this issue can be described as (6)

$$
\text { Fitness_value }(\text { M_best })=\min \left(A F \_\max \right) \text {. }
$$

$A F \_$max is the maximum value of $A F$ corresponding to given coding matrix.

After 3000 times of iteration, the results of optimization are shown in Fig. 8. It can be seen that fitness value declines rapidly during the first few iterations and tends to become steady after 800 times of iteration. Compared with the initial coding matrix and its corresponding $A F$ max, the $A F$ max corresponding to the optimized coding matrix can be suppressed significantly, as shown in Fig. 8 and Fig. 9.

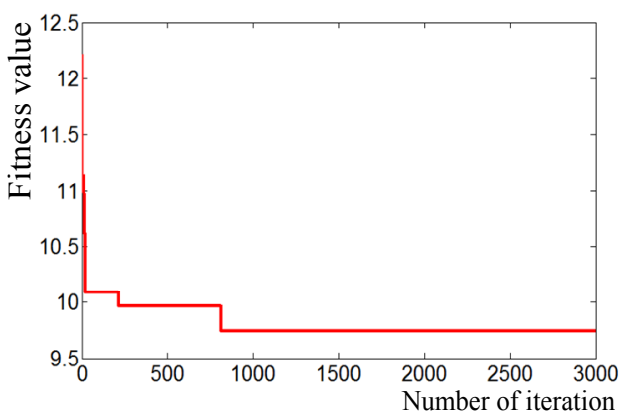

Fig. 8. Fitness evolution curve of optimization schedule.

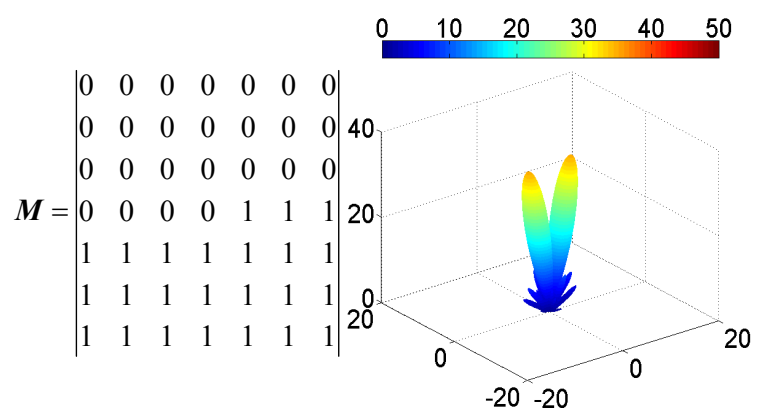

(a)

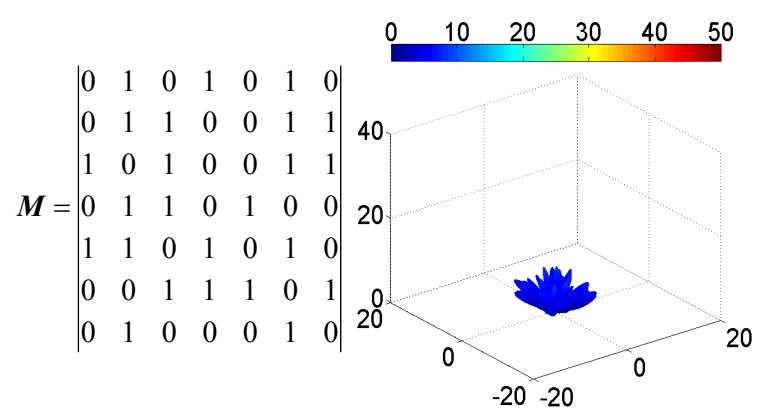

(b)

Fig. 9. (a) Initial coding matrix and its corresponding scattered far-field pattern. (b) Optimized coding matrix and its corresponding scattered far-field pattern.

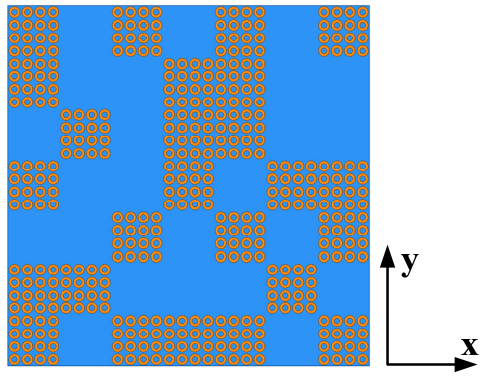

Fig. 10. The final design of optimized coding metamaterial.

Based on this optimized coding matrix, the coding metasurface can be designed as follows.

\section{Simulation and Measurement}

Simulations of the conventional chessboard surface and the proposed coding metasurface are presented and compared in this section using Ansoft HFSS, and the measurement result of fabricated coding metasurface is given in this section as well. The bistatic RCS patterns also are discussed in detail in this section.

As illustrated in Fig. 10, the substrate has the dielectric constant of 2.65 and a thickness of $3 \mathrm{~mm}$, and the overall dimension is $280 \mathrm{~mm} \times 280 \mathrm{~mm}\left(7.5 \lambda_{0} \times 7.5 \lambda_{0}\right)$. A conventional chessboard surface with same size is also simulated to compare with the coding metasurface, and the chessboard surface is designed as follows.

Figure 12(a) shows the boresight bistatic RCS of bare PEC plate, chessboard surface and coding metasurface for normally incident EM waves. Figure 12(b) shows the specular bistatic RCS of bare PEC plate, chessboard surface and coding metasurface for obliquely incident EM waves. The incident angle of obliquely incident waves is $\varphi=45^{\circ}$, $\theta=21^{\circ}$.

It can be concluded from Fig. 12(a) that the backward scattered of a metal plate can be significantly reduced by covering it with the proposed coding metasurface. As demonstrated in Fig. 12(a), the boresight RCS is suppressed by at least $7 \mathrm{~dB}$ from $8.7 \mathrm{GHz}$ to $11.3 \mathrm{GHz}$ and by more than $10 \mathrm{~dB}$ from $7 \mathrm{GHz}$ to $8.7 \mathrm{GHz}$ and from $11.3 \mathrm{GHz}$ to $20 \mathrm{GHz}$ for both $\mathrm{x}$ - and y-polarized incidences, respectively. The boresight RCS reduction of the proposed coding metasurface is better than that of conventional

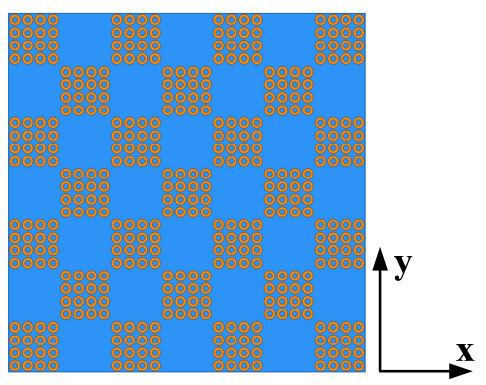

Fig. 11. The design of chessboard surface. 
chessboard. Thus, the simulated RCS reduction bandwidth of the coding metasurface is $96.6 \%$. Figure 12 (b) indicates that compared with the metal with the same dimensions, the specular RCS of the coding metasurface and chessboard surface can be significantly reduced under obliquely incident waves as well.

The simulated 3-D bistatic far-field patterns of chessboard and proposed coding metasurface at different frequencies for normally and obliquely incident waves are illustrated in Fig. 13.

From Fig. 12 and Fig. 13, it can be concluded that the main lobe of chessboard is scattered into four lobes, while that of the coding metasurface is scattered into lobes in all directions. Compared with the PEC structure with one single main beam, these two structures of chessboard and coding metasurface can achieve RCS reduction dramatically for both normally incident waves and obliquely incident waves, but the RCS reduction of coding metasurface is much better because it can redirect the normally incident EM waves in all directions equally. For bistatic detection, the strong lobes of chessboard surface can be easily detected, while the coding metasurface can redirect the incidence into all directions so that it has no distinctly strong lobes.

In order to further verify the design of this paper, a sample of the coding metasurface with the dimensions of $280 \mathrm{~mm} \times 280 \mathrm{~mm}\left(7.5 \lambda_{0} \times 7.5 \lambda_{0}\right)$ is fabricated, as depicted in Fig. 14. Both the scattered coefficients of bare PEC plate and the coding metasurface are measured to obtain the RCS reduction. The measurement setup is depicted in Fig. 15.

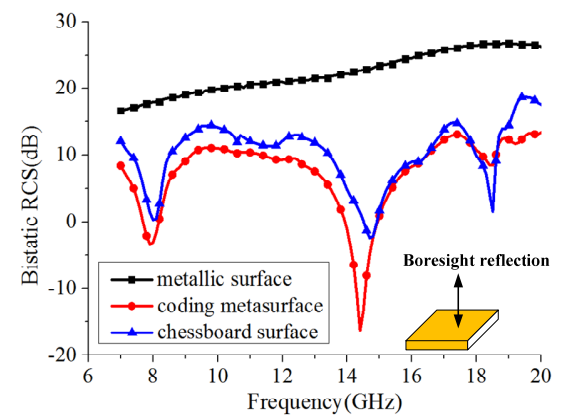

(a)

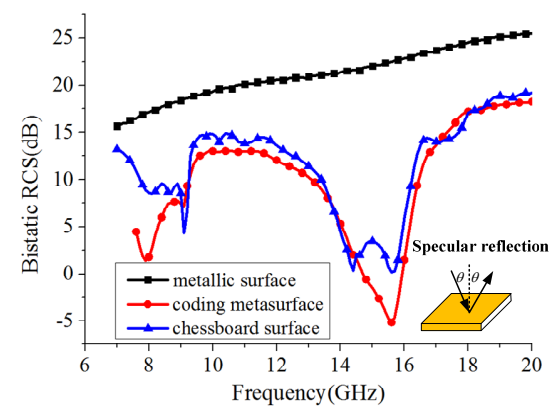

(b)

Fig. 12. The simulated bistatic RCS for chessboard, coding and metallic surfaces under (a) normally incident waves and (b) obliquely incident waves.
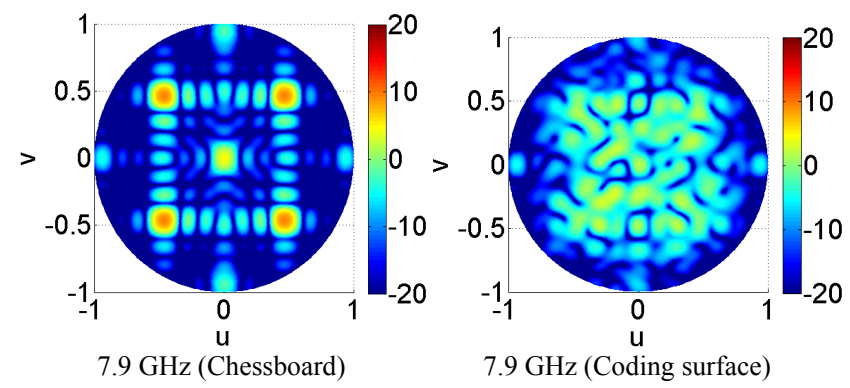

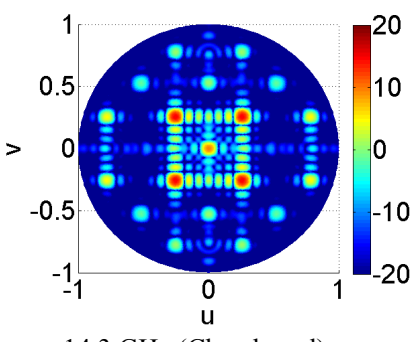

14.3 GHz (Chessboard)

(a)

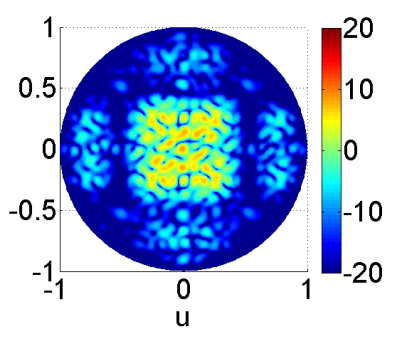

14.3 GHz (Coding surface)

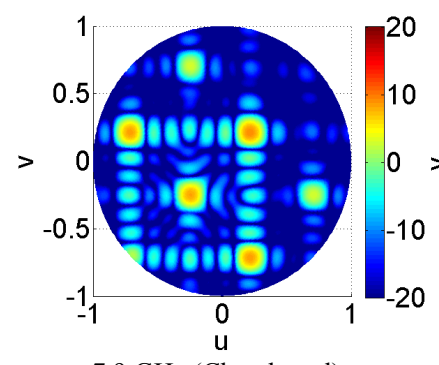

7.9 GHz (Chessboard)

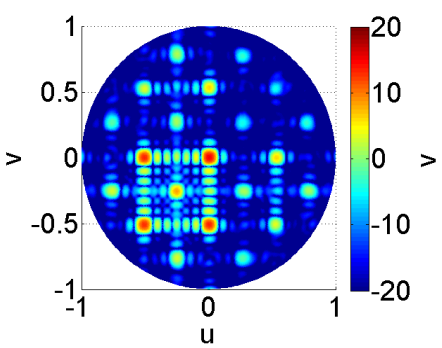

14.3 GHz (Chessboard)

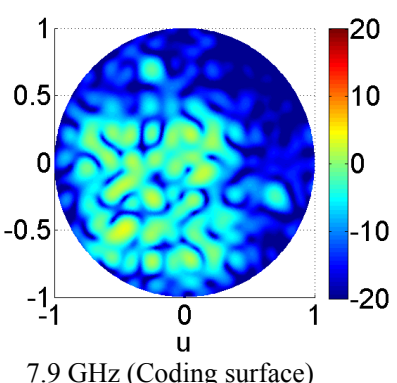

$7.9 \mathrm{GHz}$ (Coding surface)

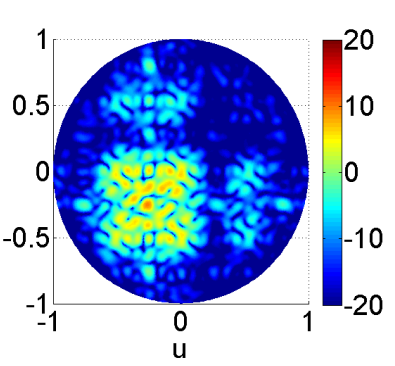

14.3 GHz (Coding surface) (b)

Fig. 13. Simulated 3-D bistatic scattered far-field patterns of chessboard and coding metasurface at different frequency points under (a) normally incident EM waves and (b) obliquely incident EM waves (the incident angle is $\varphi=45^{\circ}, \theta=21^{\circ}$ ).

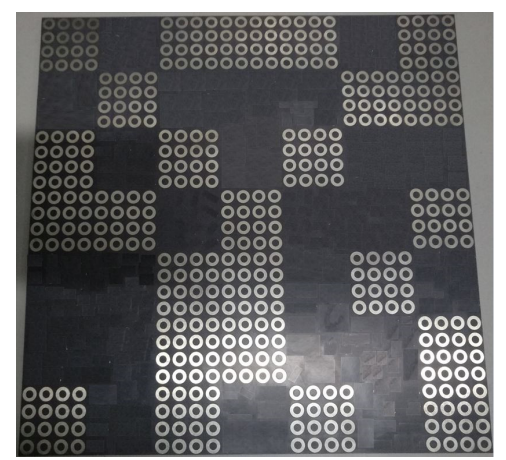

Fig. 14. Fabricated sample of coding metasurface. 


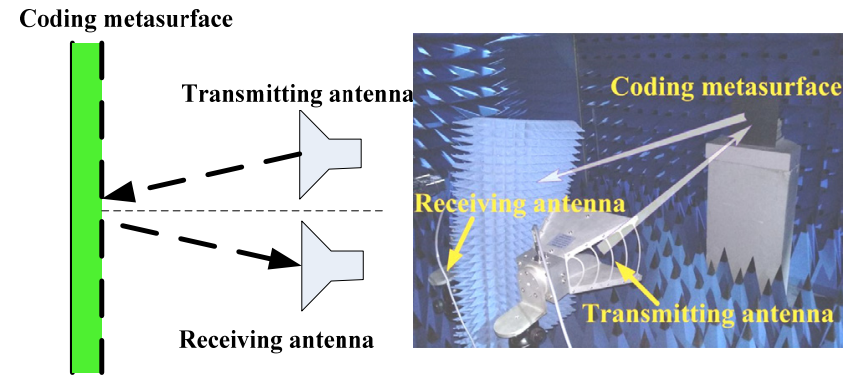

Fig. 15. Measurement setup.

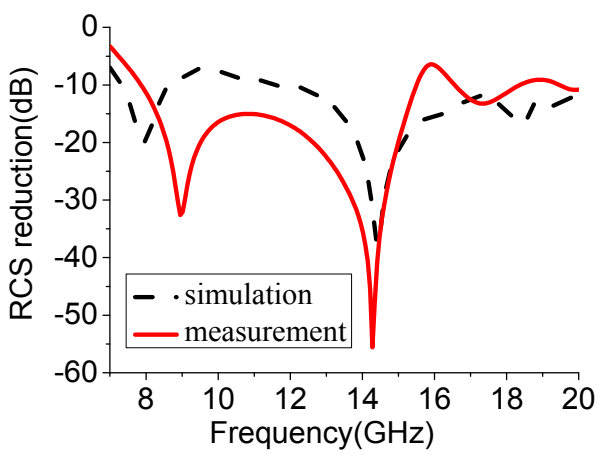

Fig. 16. The comparison of boresight RCS reduction between simulated and measured results of coding metasurface under normally incident waves.

In Fig. 15, two antennas are connected to a vector network analyzer.

As expected, the RCS of the proposed coding metasurface can be reduced dramatically in the frequency band of $7 \mathrm{GHz}-20 \mathrm{GHz}$, compared with that of PEC plate. The experimental result is consistent well with that of simulation. Although the RCS reduction of coding metasurface in the frequency band of $8.7 \mathrm{GHz}-11.3 \mathrm{GHz}$ is a little less, the proposed coding metasurface still performs well in the operation bandwidth.

\section{Conclusion}

Two novel AMC structures, composed of circular loop patch and substrate, are designed, and they are simulated using Ansoft HFSS. These two structures can achieve 180 degrees of phase difference, so these two structures are encoded as unit " 0 " and unit " 1 ". A coding matrix is introduced in this paper to describe the coding sequences of coding metasurface. Using Simulated Annealing algorithm, an optimized coding matrix which can redirect normally incident EM waves into all directions is achieved in this paper. Based on the optimized coding matrix, a coding metasurface composed of these two structures is designed and simulated, and the conventional chessboard is also simulated as well. Compared with bare PEC plate, the RCS of chessboard and coding metasurfaces can be reduced, and the RCS reduction of coding metasurface is much better.

It is verified that a wideband RCS reduction with bandwidth of $96.3 \%$ is obtained by optimizing the coding matrix of coding metasurface. Because the scattered fields of coding metasurface are redirected in all directions, the RCS can be reduced, and it has been verified with the simulation and measurement results.

\section{Acknowledgments}

Authors thank the supports from the National Natural Science Foundation of China under Grant (No.61271100, No.61471389, and No.61671464)

\section{References}

[1] SHELBY, R. A., SMITH, D. R., SCHULTZ, S. Experimental verification of a negative index of refraction. Science, 2001, vol. 292, no. 6, p. 77-79. DOI: $10.1126 /$ science. 1058847

[2] GLYBOVSKi, S. B., TRETYAKOV, S. A., BELOV, P. A., et al. Metasurfaces: From microwaves to visible. Physics Reports, 2016, vol. 634, p. 1-72. DOI: 10.1016/j.physrep.2016.04.004

[3] EPSTEIN, A., ELEFTHERIADES, G. V. Huygens' metasurfaces via the equivalence principle: design and applications. Journal of the Optical Society of America B, 2015, vol. 33, no. 2, p. A31-A50. DOI: 10.1364/JOSAB.33.000A31

[4] YU, N. F., GENEVET, P., KATS, M. A., et al. Light propagation with phase discontinuities: Generalized laws of reflection and refraction. Science, 2011, vol. 334, no. 6054, p. 333-337. DOI: 10.1126/science. 1210713

[5] RADI, Y., ASADCHY, V. S., TRETYAKOV, S. A. Tailoring reflections from thin composite metamirrors. IEEE Transactions on Antennas and Propagation, 2014, vol. 62, no. 7, p. 3749-3760. DOI: 10.1109/TAP.2014.2319851

[6] PFEIFFER, C., GRBIC, A. Metamaterial Huygens' surfaces: Tailoring wave fronts with reflectionless sheets. Physical Review Letters, 2013, vol. 110, no. $19 . \quad$ DOI: 10.1103/PhysRevLett.110.197401

[7] LI, S. J., CAO, X. Y, XU, L. M., et al. Ultra-broadband Reflective Metamaterial with RCS Reduction based on Polarization Convertor, Information Entropy Theory and Genetic Optimization Algorithm. Scientific Reports, 2016, vol. 6, p. 37409. DOI: 10.1038/srep37409

[8] TRETYAKOV, S. A. Metasurfaces for general transformations of electromagnetic fields. Philosophical Transactions of the Royal Society A, 2015, vol. 373. DOI: 10.1098/rsta.2014.0362

[9] LANDY, N. I., S. SAJUYIGBE, J. J. MOCK, et al. A perfect metamaterial absorber. Physical Review Letters, 2008, vol. 100, no. 20. DOI: 10.1103/PhysRevlett.100.207402

[10] SU, P., ZHAO, Y. J., JIA, S. L., et al. An ultra-wideband and polarization-independent metamaterial for RCS reduction. Scientific Report, 2016, vol. 6. DOI: 10.1038/srep20387

[11] CHEN, W. G., BALANIS, C. A., BIRTCHER, C. R. Checkerboard EBG surfaces for wideband radar cross section reduction. IEEE Transactions on Antennas and Propagation, 2015, vol. 63, no. 6, p. 2636-2645. DOI: 10.1109/TAP.2015.2414440

[12] CHEN, W. G., BALANIS, C. A., BIRTCHER, C. R. Dual wideband checkerboard surfaces for radar cross section reduction. IEEE Transactions on Antennas and Propagation, 2016, vol. 64, no. 9, p. 4133-4138. DOI: 10.1109/TAP.2016.2583505 
[13] ESMAELI, S. H., SEDIGHY, S. H. Wideband radar cross-section reduction by AMC. Electronics Letters, 2016, vol. 52, no. 1, p. $70-71$. DOI: $10.1049 / \mathrm{el} .2015 .3515$

[14] PANQUAY, M., IRIARTE, J. C., EDERRA, I., et al. Thin AMC structure for radar cross-section reduction. IEEE Transactions on Antennas and Propagation, 2007, vol. 55, no. 12, p. 3630-3638. DOI: 10.1109/TAP.2007.910306

[15] LIU, S., CUI, T, J., XU, Q. Anisotropic coding metamaterials and their powerful manipulation of differently polarized terahertz waves. Light: Science \& Applications, 2016, vol. 5, e16076. DOI: $10.1038 / 1 \mathrm{sa} .2016 .76$

[16] LIU, S., CUI, T. J. Flexible controls of terahertz waves using coding and programmable metasurfaces. IEEE Journal of Selected Topics in Quantum Electronics, 2017, vol. 23, no. 4, p. 1-12. DOI: 10.1109/JSTQE.2016.2599273

[17] CUI, T. J. Information entropy of coding metasurface. Light: Science \& Applications, 2016, vol. 5, e16172. DOI: 10.1038/1sa.2016.172.

[18] LIU, S., NOOR, A., DU, L. L., et al. Anomalous refraction and nondiffractive Bessel-beam generation of terahertz waves through transmission-type coding metasurfaces. ACS Photonics, 2016, vol. 3, no. 10, p. 1968-1977. DOI: 10.1021/acsphotonics.6b00515

[19] LIU, S., ZHANG, L., YANG, Q. L., et al. Frequency-dependent dual-functional coding metasurfaces at terahertz frequencies. Advanced Optical Materials, 2016. DOI: 10.1002/adom.201600471

[20] ZHAO, Y., CAO, X. Y., GAO, J., et al. Broadband diffusion metasurface based on a single anisotropic element and optimized by the Simulated Annealing algorithm. Scientific Reports, 2016, vol. 6. DOI: $10.1038 / \mathrm{srep} 23896$

[21] BALANIS, C. A. Antenna Theory: Analysis and Design. 3rd ed. Hoboken (NJ, USA): Wiley, 2005. ISBN: 978-0471667827

\section{About the Authors ...}

Liao-ri JI-DI was born in Sichuan province, China. He received his B.S. degree from Electronic and Information College, Tongji University in 2016, and he was the commencement student speaker of Tongji University in 2016.
He currently works towards his M.S. degree. In his research, he specializes in metamaterial, antenna array design and RCS reduction techniques.

Xiang-yu CAO received her M.S. degree from the Air Force Missile Institute in 1989. In the same year, she joined the Air Force Missile Institute. She received her Ph.D. degree in the Missile Institute of AFEU in 1999. From 1999 to 2002, she was engaged in postdoctoral research in Xidian University, China. She was a Senior Research Associate in the Dept. of Electronic Engineering, City University of Hong Kong from June 2002 to Dec. 2003. She is currently a professor and a senior member of IEEE. Her research interests include computational electromagnetic, electromagnetic metamaterials and their antenna applications.

Yao TANG was born in Shandong province, China. He received his B.S. degree from Shandong University of Technology in 2009. He currently works towards his M.S. degree. His research interests are electromagnetic metamaterials and their antenna applications.

Si-ming WANG was born in Zhejiang province, China. She received her B.S. degree from AFEU in 2016. She currently works towards her M.S. degree. Her research interests are antenna design and electromagnetic metamaterials.

Yi ZHAO was born in Henan province in 1988 . He received the B.S. and M.S. degrees from the Information and Navigation Institute, Air Force Engineering University of CPLA, China, in 2011 and 2013, respectively. His research interest is in electromagnetic metamaterials and their antenna applications.

Xue-wen ZHU received his B.S. degree from AFEU in 2016. He currently works towards his M.S. degree. His research interests are antenna design and electromagnetic metamaterials. 